\title{
Preparation and Characterisation of Collagen from Freshwater Fish Scales
}

\author{
Fengxiang Zhang ${ }^{{ }^{*}}$, Anning Wang ${ }^{2}$, Zhihua $\mathrm{Li}^{1}$, Shengwen $\mathrm{He}^{1}$, Lijun Shao ${ }^{1}$ \\ ${ }^{1}$ Collage of Public Health, Weifang Medical University, Weifang, China; ${ }^{2}$ Weifang People's Hospital (Brain Hospital), Weifang, \\ China. \\ Email: ${ }^{*}$ zfx0515@163.com
}

Received May 23 $3^{\text {rd }}, 2011$; revised July $31^{\text {st }}, 2011$; accepted August $7^{\text {th }}, 2011$.

\begin{abstract}
Acid-soluble collagen (ASC) and pepsin-solubilized collagen (PSC) were prepared from the waste freshwater carp fish scales. The results of SDS-PAGE showed that purified collagens were composed of at least two different chains which were in accordance with the type I collagen with $\alpha$ chain composition of $\left(\alpha_{1}\right)_{2} \alpha_{2}$. Compared with the carp fish ordinary muscle type I collagen, porcine dermis type I collagen and other seawater fish collagens, freshwater carp fish scales collagen contained relative high half-cystine $(C y s-s)$, but lower denaturation temperature $(T d)$ than the porcine dermis type I collagen. These collagens had evident absorption at $230 \mathrm{~nm}$ by UV-Vis spectra. The spectrum X-ray diffraction showed that the collagen remained single-helix and tri-helix configuration with the minimum values of the repeat spacings (d) of about 4.48 A and 11.87 A. Therefore, to make more effective use of limited-resources, carp fish scales can be a potential resource for the extraction of type I collagen or gelatin.
\end{abstract}

Keywords: Carp Fish Scale, ASC (Acid-Solubilized Collagens), PSC (Pepsin-Solubilized Collagens), Type I Collagen

\section{Introduction}

Collagen is an important protein in the organisms, and the use of collagen in industry for health foods, cosmetics, and biomaterials is expanding. At present, because of the outbreak of bovine spongiform encephalopathy (BSE) and the foot-and-mouth disease (FMD), the main sources of collagen in many fields are limited to those of bovine or porcine dermis, so more and more studies turn to find safe sources of collagen. There have been many reports of collagen in aquatic animals.

Fish scales are biocomposites of highly ordered type I collagen fibers and hydroxyapatite $\mathrm{Ca}_{10}(\mathrm{OH})_{2}\left(\mathrm{PO}_{4}\right)_{6}[1-3]$. Collagens extracted from fish scales of black drum, sheep's head sea bream, Red seabream, Red Tilapia, sardine, Japanese sea-bass, skipjack tuna, ayu, yellow sea bream and horse mackerel have been reported [4-6]. These collagens are mainly type I collagen with a lower denaturation temperatures than the collagen of porcine dermis. These fish are mostly seawater fish and there have been fewer reports about the freshwater fish scales. Carp fish (Cyprinus carpio) is one of the primary fish species in freshwater breeding industry in China. During the processes of fish, a great amount of fish scales are dumped, which is a great waste because the scales of carp fish contain a large amount of collagen. Therefore in this paper, we extracted and partially characterized the collagens of carp fish scale for potential utilization.

\section{Materials and Methods}

\subsection{Materials}

Fresh carp fish scales were collected from market during the month of October. The scales were washed thoroughly with distilled water, and stored at $-25^{\circ} \mathrm{C}$ until used. All reagents used were of analytical grade.

\subsection{Demineralization Process}

Initially, the fish scales were washed twice in $10 \mathrm{wt} \%$ of $\mathrm{NaCl}$ solutions to remove unnecessary proteins on the surface by stirring the solution for $24 \mathrm{~h}$. Demineralization was achieved with $0.4 \mathrm{~mol} / \mathrm{l} \mathrm{HCl}$ solution (dry scales: solution $=1: 15$ ) for $90 \mathrm{~min}$. The demineralized scales were washed three times with distilled water for collagen extraction.

\subsection{Morphological Analysis}

Morphological analysis was undertaken using a QUANTA-200 SEM (FEI Company, USA) at an accelerating voltage of $10 \mathrm{kV}$. The perpendicular cut surfaces of the fresh scales and the demineralized scales were coated 
with platinum for morphological observations.

\subsection{Extraction of Acid- and Pepsin-Solubilized Collagens (ASC and PSC)}

All the preparative procedures were performed at $4{ }^{\circ} \mathrm{C}$. Carp fish scales were extracted with $0.5 \mathrm{M}$ acetic acid for 2 days, and the extracts were centrifuged at $10,000 \times \mathrm{g}$ for $30 \mathrm{~min}$. The residues were re-extracted with the same solution for 1 day, and these extracts were centrifuged under the same conditions. The supernatants were combined and salted out by adding $\mathrm{NaCl}$ to a final concentration of $0.7 \mathrm{M}$. The precipitated collagens were separated by centrifugation at $10,000 \times \mathrm{g}$ for $30 \mathrm{~min}$ and redissolved in $0.5 \mathrm{M}$ acetic acid to precipitate with $\mathrm{NaCl}$ again. The resultant precipitates were dialyzed against distilled water and lyophilized. The residues from the acetic acid extraction were suspended in $0.5 \mathrm{M}$ acetic acid and digested with $0.5 \%(\mathrm{w} / \mathrm{v})$ pepsin (Sigma p7000) for $72 \mathrm{~h}$. The extraction steps of PSC were the same as the extraction of ASC.

\subsection{Amino Acid Analysis}

Collagen samples were hydrolyzed under reduced pressure in $6 \mathrm{M} \mathrm{HCI}$ at $110^{\circ} \mathrm{C}$ for $22 \mathrm{~h}$, and the hydrolysates were analyzed by an amino acid analyzer HP1100 (Agilent, USA).

\subsection{SDS-Polyacrylamide Gel Electrophoresis (SDS-PAGE)}

SDS-PAGE was performed according to the methods of Laemmli [7] using 5\% stacking gel and 7.5\% resolving gel. The samples were dissolved in $0.6 \mathrm{M}$ Tris- $\mathrm{HCl}$ buffer $(\mathrm{pH}$ 6.8) which contained $25 \%$ (v/v) glycerine, $2 \%(\mathrm{w} / \mathrm{v})$ SDS, $5 \%(\mathrm{v} / \mathrm{v}) \beta$-mercaptoethanol, and $0.1 \%$ (w/v) Bromophenol blue. After electrophoresis, gels were visualized with Coomassie Brilliant Blue R-250.

\subsection{Determination of Denaturation Temperature}

The denaturation temperature was measured according the method of Nagai et al. [8]. Ten $\mathrm{mL}$ of the $0.03 \%$ collagen solution in $0.1 \mathrm{M}$ acetic acid were used for the viscosity measurement, and measurement was done using an Ostwald viscometer. The thermal determination curve was obtained by measuring solution viscosity at several temperatures fro$\mathrm{m} 10^{\circ} \mathrm{C}$ to $50^{\circ} \mathrm{C}$; the temperature was raised stepwise and maintained for 30 mins at each point. The denaturation temperature (Td) was determined as the temperature at which the change in viscosity was half completed.

\subsection{X-Ray Diffraction Analysis}

X-ray diffraction pattern of the PSC was obtained using $\mathrm{CuKa}$ radiation from a rotating anode generator operated at $35 \mathrm{kV}$ and $20 \mathrm{~mA}$ in the range of $2 \theta=5^{\circ}-50^{\circ}$ with Mono-single filter (D8 ADVANCE, Bruker axs, German).
The scanning step was 0.02 degree while the scanning speed was 2 degree/min.

\subsection{UV-Vis Spectra}

The UV-Vis absorption spectrum of PSC collagen was recorded using a Shimadzu spectrophotometer UV-240 in the range of $200-400 \mathrm{~nm}$.

\section{Results and Discussion}

\subsection{Morphological Analysis of Carp Fish Scale}

The scales of teleost fish were composed of calciumdeficient hydroxyapatite $\mathrm{Ca}_{10}(\mathrm{OH})_{2}\left(\mathrm{PO}_{4}\right)_{6}$ and extracellular matrix, mainly type I collagen fibers, which together formed a highly ordered three-dimensional structure. Each scale consisted of two distinct regions: an external (osseous) layer and an internal fibrillary plate [1]. Needlelike or flaky crystals of apatite in random orientation were observed in the outer layer [9]. As calcifycation proceeded, the crystals of apatite penetrated into the internal fibrillary plate overlap zone and compressed the tri-helix collagen molecules (Figure 1(a)). So in order to get the collagen, the fish scales first should be demineralized. Figure 1(b) showed the structure of the demineralized carp scale. Because of demineralization, the plywood-like structures were exposed thus making it easier to dissolve collagen by acid solution. The growth rings from which the fish growth age can be determined were showed on the surface of the carp fish scale, Figure 1(c).

\subsection{Subunit Composition}

Subunit compositions of these extracted collagens were examined by SDS-PAGE (Figure 2). Both the PSC and ASC were shown to contain at least two different $\alpha$ chains. Together with the results of precipitation properties by $\mathrm{NaCl}$ under acidic $\mathrm{pH}$ and SDS-PAGE, collagen of carp fish scale might be classified as type I collagen, therefore, the two $\alpha$ chains were $\alpha_{1}$ and $\alpha_{2}$ with the molecular weight approximating $117.3 \mathrm{KDa}$ and 107.4 KDa. The relative staining intensity of the $\alpha_{1}$ to $\alpha_{2}$ was stronger, consequently the amount of $\alpha_{1}$ was more than $\alpha_{2}$; A fish-specific chain, $\alpha_{3}$ of type I collagen, was found in teleostean fish skin [10], and the $\alpha_{3}$ gene appeared to have arisen from a duplication of the $\alpha_{1}$ gene near the time of the adaptive radiation of bony fish [11], but there are no report about the $\alpha_{3}$ chain found in fish scale collagen. In that study, it was not separated from the corresponding $\alpha 1$ chain under these electrophoretic conditions. On the other hand, the SDS-PAGE showed a single $\alpha 1$ band with relative higher staining intensity, so the constitution of the trimmer of carp fish scale type I collagen was deduced to be $\left(\alpha_{1}\right)_{2} \alpha_{2}$. Moreover, a small amount of the $\beta$ chain which was dimerized by $\alpha$ component was obtained 


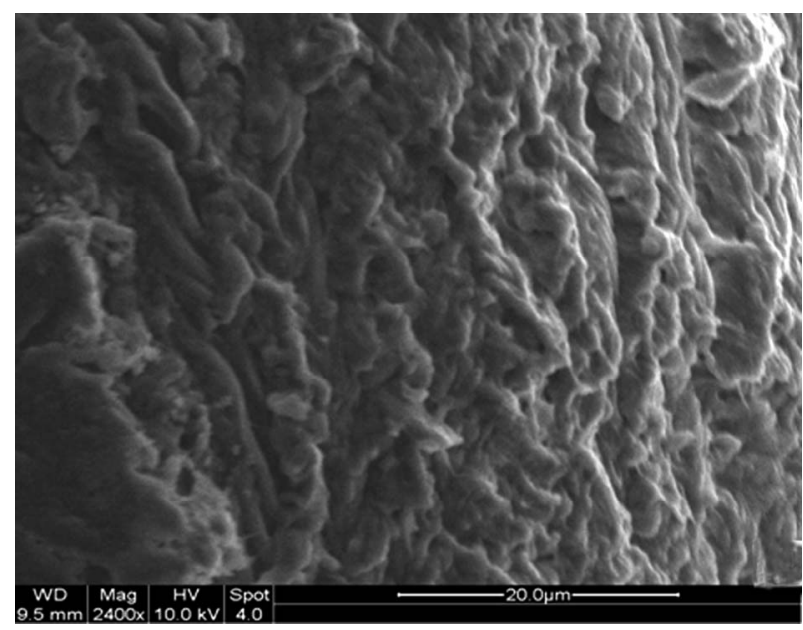

(a)

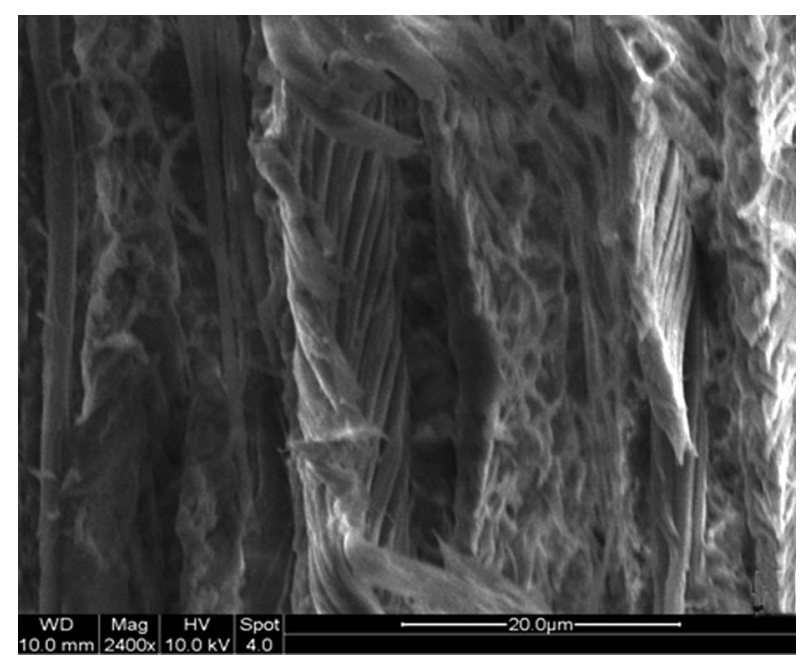

(b)

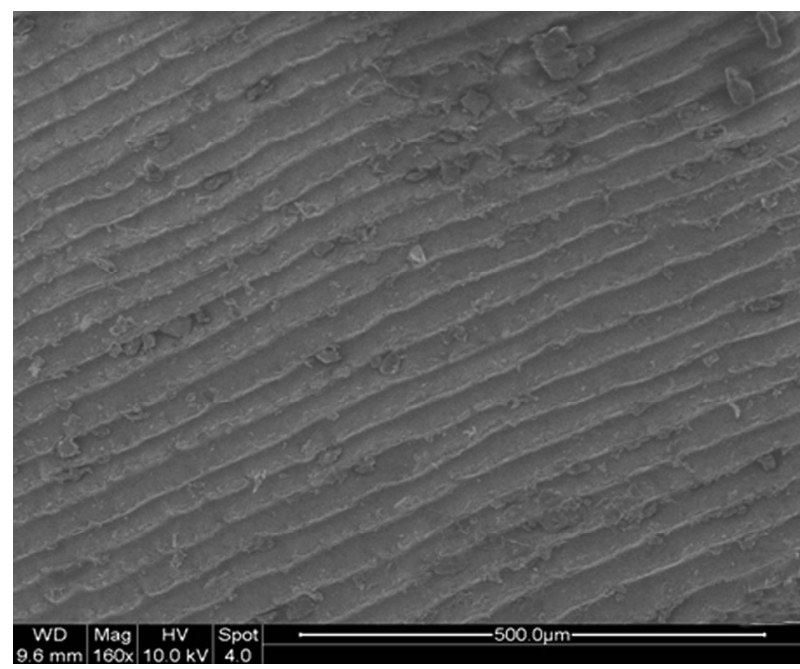

(c)

Figure 1. SEM micrograph of the perpendicular cut surfaces of the fresh carp scale (a) the demineralized carp scale (b) and the growth rings on the surface of the carp fish scale(c).

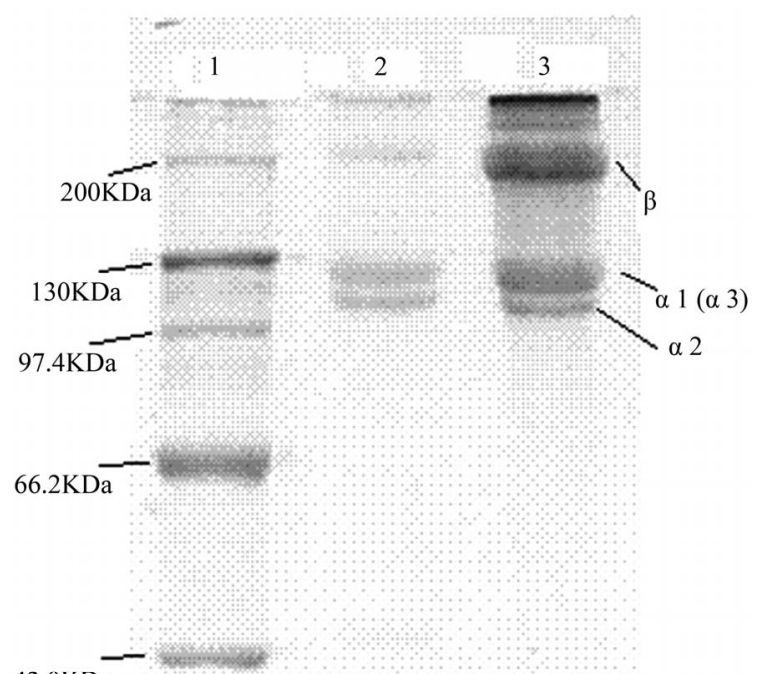

Figure 2. SDS-polyacrylamide gel electrophoresis of extracted collagen and high molecular weight markers. Lane1, high molecular weight markers; Lane2, the extracted collagen of PSC; Lane3, the extracted collagen of ASC.

in these collagens, and the content of $\beta$ chain was less in PSC than in ASC because of the hydrolysis by pepsin.

\subsection{Amino Acid Composition}

The amino compositions per 1000 total residues of carp fish scale acid-soluble (ASC) and pepsin-soluble collagen (PSC) were showed in Table 1. The collagens were high in proline (Pro), glycine (Gly), and hydroxyproline (Hyp), which were due to the characteristic (GlyPro-Hyp)n, triple helical repeat of all collagens. High levels of alanine (Ala), as observed in collagens from animal species, were also measured in the fish scale collagens. Except Cys-s, the other amino acid compositions of carp fish scale acid-soluble and pepsinsoluble collagens were similar to that of carp fish ordinary muscle type I collagen [12] and porcine dermis type I collagen [5]. The freshwater fish scales contained relative high Cys-s, while there was almost no Cys-s detected in other seawater fish collagens [4, 13-16]. The degrees of hydroxylation of proline for carp fish scale collagen were $47.2 \%$ (PSC) and 44.7\% (ASC), which were likely to affect the stability of the collagen fibers and denaturation temperatures $[5,14]$. The results of the 
Table 1. Amino acid composition of fish scale pepsin-solubilized collagen (PSC) and acid-solubilized collagen (ASC), residues/1000.

\begin{tabular}{|c|c|c|c|c|}
\hline Amino acid & PSC of carp fish scale & ASC of carp fish scale & $\begin{array}{l}\text { Type I collagen of carp } \\
\text { fish ordinary Muscle }^{\text {a }}\end{array}$ & Type I collagen of porcine dermis ${ }^{b}$ \\
\hline Asp & 46 & 49 & 41 & 44 \\
\hline Glu & 74 & 77 & 72 & 72 \\
\hline Ser & 35 & 38 & 37 & 33 \\
\hline His & 5 & 6 & 5 & 5 \\
\hline Gly & 302 & 306 & 339 & 341 \\
\hline Thr & 22 & 21 & 26 & 16 \\
\hline $\operatorname{Arg}$ & 48 & 50 & 52 & 48 \\
\hline Ala & 114 & 117 & 121 & 115 \\
\hline Tyr & 22 & 21 & 3 & 1 \\
\hline Cys-s & 30 & 32 & - & - \\
\hline Val & 18 & 19 & 16 & 22 \\
\hline Met & 10 & 12 & 12 & 6 \\
\hline Phe & 13 & 15 & 13 & 12 \\
\hline Ile & 10 & 12 & 10 & 10 \\
\hline Leu & 22 & 24 & 22 & 22 \\
\hline Lys & 25 & 26 & 26 & 27 \\
\hline Ho-pro & 109 & 89 & 85 & 97 \\
\hline Pro & 122 & 110 & 112 & 123 \\
\hline
\end{tabular}

a: Sato et al., 1988. b: Ikoma et al., 2003.

electrophoretic migration and amino acid composition suggested that the chemical compositions of type I collagen were highly conserved among the scales.

\subsection{Denaturation Temperature}

The denaturation temperature (Td) of carp fish scale collagen sample was calculated from the thermal denaturetion curves, Figure 3, and the Td of the collagen was taken to be the temperature at which the fractional viscosity was 0.5 . Td of the ASC of carp fish scale was about $32.9^{\circ} \mathrm{C}$, but the Td of PSC of carp fish scale was only about $29.0^{\circ} \mathrm{C}$, which was mainly because of the enzyme hydrolysis action. The Td of the ASC of carp fish scale was lower than the porcine skin collagen but higher than the Td of many cold-water fish collagens measured under the same conditions [6, 17, 18]. Earlier studies [19] showed that the stability of collagen is correlated to environmental and body temperatures, but present studies have reported that Hydroxyproline is important in maintaining the stability of trimmers in collagen $[5,14]$.

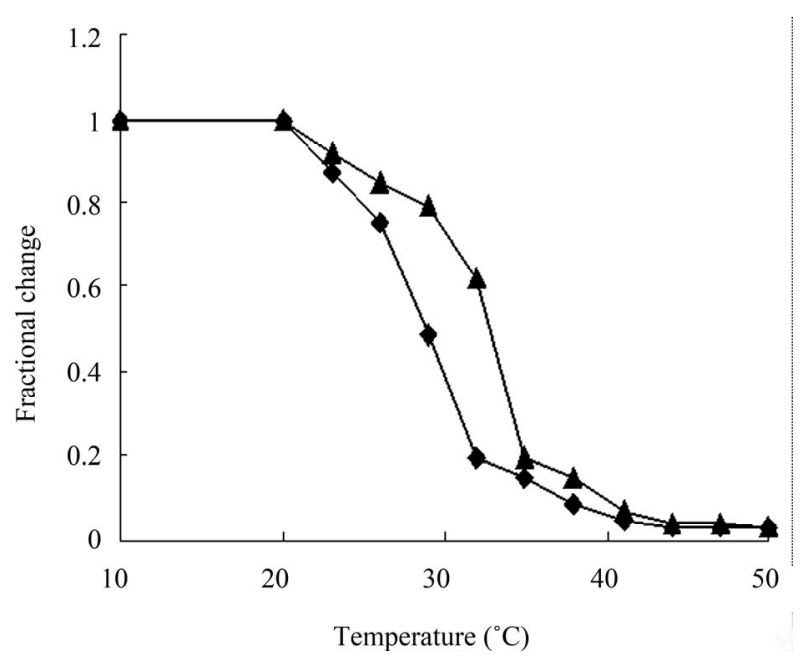

Figure 3. Thermal denaturation curve of carp fish scale collagen solution as measured by viscosity in $0.1 \mathrm{M}$ acetic acid. The incubation time at each temperature was $30 \mathrm{~min}$. Collagen concentration: $0.03 \% . \Delta$ : acid-solubilized collagen (ASC) of the carp fish scale, : (pepsin-solubilized collagen)of the carp fish scale. 


\subsection{UV-Vis Spectra}

Phenylalanine and tyrosine have absorption bands between 250 and $290 \mathrm{~nm}$, but the PSC collagen of carp fish scale had a distinct absorption near $233 \mathrm{~nm}$ (Figure 4), closer to the absorption of channel catfish skin collagen [20], which was in accordance with the characteristic absorption of collagen.

\subsection{X-Ray Diffraction Analysis}

$\mathrm{X}$-ray diffraction was used to investigate the collagen fibril distribution and orientation in mineralized tissues [21]. Figure 5 showed the X-ray spectrum of the lyophilized pepsin-solubilized carp fish scale collagen crystal. There were two diffraction peaks at the diffraction angles $(2 \theta)$ about $7.44^{\circ}$ and $19.78^{\circ}$. The first one was sharp but the second one was wide, which were in accordance with the characteristic diffraction peaks of collagen. The diffraction peaks showed that this protein had ordered structure or ordered structure snippet [22]. From the Bragg equation $\mathrm{d}(\AA)=\lambda / 2 \sin \theta(\lambda=1.54 \AA)$, the minimum values $(d)$ of the repeat spacings were calculated. The $d$ of the sharp peak was11.87 $\AA$ and that of the wide peak was $4.48 \AA$, which were related to the diameter of the tri-helix collagen molecule and the single left-hand helix chain. The collagen extracted had a tri-helix structure.

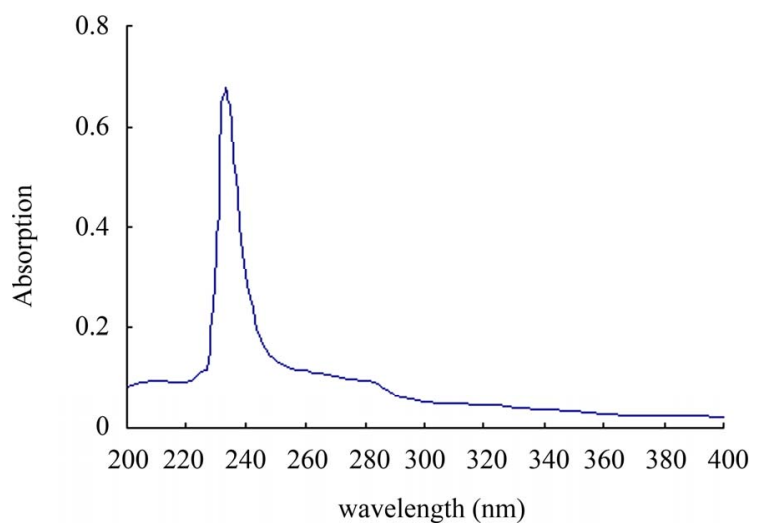

Figure 4. UV-Vis spectra of pepsin-solubilized carp fish scale collagen.

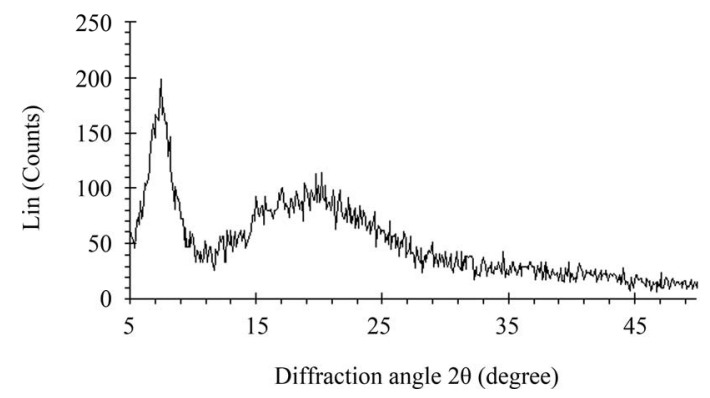

Figure 5. X-ray spectrum of pepsin-solubilized carp fish scale collagen.

\section{Conclusions}

In this study, the carp fish scale collagens were extracted by $0.5 \mathrm{M}$ acetic acid or pepsin. This collagen had abundant amino composition, but lower denaturation temperature compared to the porcine dermis collagen. The SDS-PAGE and X-ray diffraction analysis indicated that the collagen had tri-helix structure. It was found that a great amount of freshwater fish scales were dumped as waste, but the result showed that it is possible to use the fresh fish scales as an important collagen or gelatin source.

\section{Acknowledgements}

The authors gratefully acknowledge the financial support of Shandong Provincail Natural Science Foundation, China (Project No. ZR2010HQ044) and the Startup Foundation for Docotor of Weifang Medical University.

\section{REFERENCES}

[1] H. Onozato and N. Watabe, "Studies on Fish Scale Formation and Resorption," Cell and Tissue Research, Vol 201, No. 3, 1980, pp. 303-316. doi:10.1007/BF00236999

[2] L. Zylberberg, J. Bereiter-Hahn and J.-Y. Sire, "Cytoskeletal Organization and Collagen Orientation in the Fish Scales," Cell and Tissue Research, Vol. 253, No. 3, 1988, pp. 597-607. doi:10.1007/BF00219750

[3] T. Ikoma, H. Kobayashi, J. Tanaka, D. Walsh and S. Mann, "Microstructure, Mechanical, and Biomimetic Properties of Fishscales from Pagrus Major," Journal of Structural Biology, Vol. 142, No. 3, 2003, pp. 327-333. doi:10.1016/S1047-8477(03)00053-4

[4] M. Ogawa, R. J. Portier, M. W. Moody, J. Bell, M. A.Schexnayder and J. N. Losso, "Biochemical Properties of Bone and Scale Collagens Isolated from the Subtropical Fish Black Drum (Pogonia Cromis) and Sheepshead Seabream (Archosargus Probatocephalus)," Food Chemistry, Vol. 88, No. 4, 2004, pp. 495-501. doi:10.1016/j.foodchem.2004.02.006

[5] T. Ikoma, H. Kobayashi, J. Tanaka, D. Walsh and S. Mann, "Physical Properties of Type I Collagen Extracted from Fish Scales of Pagrus Major and Oreochromis Niloticas," International Journal of Biological Macromolecules, Vol. 32, No. 3-5, 2003, pp. 199-204. doi:10.1016/S0141-8130(03)00054-0

[6] T. Nagai, W. Worawattanamateekul, N. Suzuki, T. Nakamura, T. Ito, et al., "Isolation and Characterization of Collagen from Rhizostomous Jellyfish (Rhopilema Asamushi)," Food Chemistry, Vol. 70, No. 2, 2000, pp. 205208. doi:10.1016/S0308-8146(00)00081-9

[7] U. K. Laemmli, "Cleavage of Structural Proteins during the Assembly of the Head of Bacteriophage T4," Nature, Vol. 227, No. 5259, 1970, pp. 680-685. doi: $10.1038 / 227680 \mathrm{a} 0$

[8] T. Nagai, T. Ogawa, T. Nakamura, T. Ito, H. Nakagawa, K. Fujiki, M. Nakao and T. Yano, "Collagen of Edible 
Jellyfish Exumbrella," Journal of the Science of Food and Agriculture, Vol. 79, No. 6,1999, pp. 855-858. doi:10.1002/(SICI)1097-0010(19990501)

[9] O. P. Oslon and N. Watabe, "Studies on Formation and Resorption of Fish Scales," Cell and Tissue Research, Vol. 211, No. 2, 1980, pp. 303-316. doi:10.1007/BF00236451

[10] S. Kimura, Y. Ohno, Y. Miyauchi and N. Uchida, "Fish Skin Type I Collagen: Wide Distribution of an a3 Subunit in Teleosts," Comparative Biochemistry and Physiology, Vol. 88B, No. 1, 1987, pp. 27-34. doi:10.1016/0305-0491(92)90119-C

[11] S. Kimura, "Wide Distribution of the Skin Type I Collagen $\alpha_{3}$ Chain in Bony Fish," Comparative Biochemistry and Physiology, Vol. 102B, No. 2, 1992, pp. 255-260. doi:10.1016/0305-0491(92)90119-C

[12] K. Sato, R. Yoshinaka, M. Sato, Y. Itoh and Y. Shimizu, "Isolation of Types I and V Collagens from Carp Muscle," Comparative Biochemistry and Physiology, Vol. 90B, No. 1, 1988, pp. 155-158. doi:10.1016/0305-0491(88)90053-3

[13] M. Nishimoto, R. Sakamoto, S. Mizuta and R. Yoshinaka, "Identification and Characterization of Molecular Species of Collagen in Ordinary Muscle and Skin of the Japanese flounder Paralichthys Olivaceus," Food Chemistry, Vol. 90, No. 1-2, 2005, pp. 151-156. doi:10.1016/j.foodchem.2004.03.034

[14] J.-H. wang, S. Mizuta, Y. Yokoyama and R. Yoshinaka, "Purification and Characterization of Molecular Species of Collagen in the Skin of Skate (Raja kenojei)," Food Chemistry, Vol. 100, No. 3, 2007, pp. 921-925. doi:10.1016/j.foodchem.2005.10.046

[15] M. S. Heu, J. H. Lee, H. J. Kim, S. J. Jee, J. S. Lee, Y. J. Jeon, F. Shahidi and J.-S. Kim, "Characterization of Acid- and Pepsin-Soluble Collagens from Flatfish Skin,"
Food Science Biotechnoloy, Vol. 19, No. 1, 2010, pp. $27-$ 33. doi:10.1007/s10068-010-0004-3

[16] L. Wang, X. An, Z. Xin, L. Zaho and Q. Hu, "Isolation and Characterization of Collagen from the Skin of Deep-Sea Redfish (Sebastes Mentella)," Journal of Food Science, Vol. 72, No. 8, 2007, pp. E450-E455. doi:10.1111/j.1750-3841.2007.00478.x

[17] T. Nagai and N. Suzuki, "Isolation of Collagen from Fish Waste Material-Skin, Bone and Fins," Food Chemistry, Vol. 68, No. 3, 2000, pp. 277-281. doi:10.1016/S0308-8146(99)00188-0

[18] T. Nagai, Y. Araki and N. Suzuki, "Collagen of the Skin of Ocellate Puffer Fish (Takifugu Rubripes)," Food Chemistry, Vol. 78, No. 2, 2002, pp. 137-177. doi:10.1016/S0308-8146(01)00396-X

[19] B. J. Rigby, "Amino-Acid Composition and Thermal StaBility of the Skin Collagen of the Antarctic Ice-Fish," Nature, Vol. 219, No. 1, 1968, pp. 166-167. doi: $10.1038 / 219166 \mathrm{a} 0$

[20] H. Y. Liu, D. Li and S. D. Guo, "Studies on Collagen from the Skin of Channel Catfish (Ictalurus Punctaus)," Food Chemistry, Vol. 101, No. 2, 2007, pp. 621-625. doi:10.1016/j.foodchem.2006.01.059

[21] A. Bigi, M. Burghammer, R. Falconi, M. H. Koch, S. Panzavolta and C. Riekel, "Twisted Plywood Pattern of Collagen Fibrils in Teleost Scales: An X-ray Diffraction Investigation," Journal of Structural Biology, Vol. 136, No. 2, 2001, pp. 137-143. doi:10.1006/jsbi.2001.4426

[22] G. J. Cameron, D. E. Cairns and T. J. Wess, "The Variability in Type I Collagen Helical Pitch Is Reflected in the D Periodic Fibrillar Structure," Journal of Molecular Biology, Vol. 372, No. 4, 2007, pp. 1097-1107. doi:10.1016/i.jmb.2007.05.076 